\title{
Bulk and Surface Wettability Characteristics of Probiotic Powders in Their Compressed Disc and Packed-Bed Column Forms
}

\author{
Mohamed A. Ali, Hary L. Razafindralambo,* Giuseppina Conti, and Joël De Coninck \\ Cite This: ACS Omega 2020, 5, 22348-22355 \\ Read Online
}

ABSTRACT: Most probiotic-based products are available in powder particles under different solid-state forms. Such diversity can affect the probiotic stability, viability, and performance at different stages of processing, storage, and use. Here, we apply complementary physical chemistry techniques to characterize the bulk and surface properties of probiotic powder particles under different forms and report quantitative results of a highly concentrated multistrain reference product. The solid particle morphology, size/shape distribution, and the powder surface wettability in the compressed disc and porous packed bed forms are successively measured by sessile drop and capillary rise techniques. A complete wettability of the disc surface is observed through equilibrium contact angle measurements for various solvents, whereas the associated capillary rise data exhibit two regimes: a power law regime for the first few moments followed by a second regime, which can be described using Darcy's law. The use of this modeling approach shows the

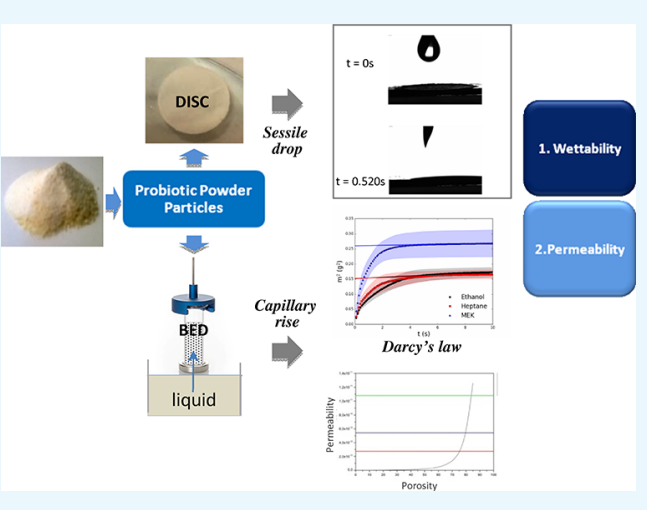
possibility of assessing the particle-packed bed permeability and porosity. These results open a new route of the structure-activity relationship study on the impact of probiotic solid particles on their functionalities and performance in promoting health benefits, related particularly to the human and animal gut permeability. This statement also strengthens the idea of using the compressed disc technique for easily performing probiotic wettability measurements.

\section{INTRODUCTION}

Nowadays, probiotics are among the most investigated research topics, ${ }^{1}$ owing to their beneficial effects on human, ${ }^{2}$ animal, ${ }^{3}$ and plant ${ }^{4}$ species. These living microorganisms are able to promote the host health by playing both preventive and therapeutic roles against numerous diseases, when they are used in adequate amounts. ${ }^{5}$ Among the most important probiotic action mechanisms include the competition for nutrients and adhesion sites with intestinal or plant pathogens, the production of metabolites (bacteriocins, organic acids, antioxidants, enzymes, and biosurfactants), and the immune system modulation. ${ }^{6}$ Moreover, probiotics may be considered as functional biocolloids, ranging from $1 \mu \mathrm{m}$ (lactic acid bacteria) to $10 \mu \mathrm{m}$ (yeasts) average particle sizes, commonly used as bioactive ingredients for food, dietary supplements, and pharmaceutical and cosmetic products under well-defined regulations. ${ }^{7}$ Most of them are available in powder particles at the solid-state or in liquid dispersed system forms. ${ }^{8}$ Consequently, they possess like any colloidal particles a couple of nano- and microscopic properties able to control their main activities and techno-functionalities. Even though much information is now available on probiotic properties (mechanisms, quality, safety, and clinical performance), a lot is still ignored, particularly those of their surface and colloidal physical chemical properties. ${ }^{9}$ However, each probiotic strain has its specific properties that govern its activities and performances in single and in mixed systems with other strains and functional components such as bulk agents, protectants, prebiotics, and so on. ${ }^{10}$

The degree of probiotic-containing products to promote health benefits depends on the capacity of the material to deliver viable functional bacteria in the adequate dose. ${ }^{11}$ Most are stored and delivered under powder particles, mainly obtained from a freeze-dried or spray-dried liquid dispersion. ${ }^{12}$ Their quality, safety, and performance depend on strains, doses, excipients, and adjuvants, which constitute the powder formulation. $^{13}$ Moreover, powder properties impact the functionality of the product, which is also dependent on the manufacturing processes, either directly through the changes in physical and chemical properties or indirectly by affecting the product stability and bacterial viability during storage. ${ }^{10}$ On one hand, the powder stability under varying temperatures and moisture content during storage is crucial. ${ }^{14}$ On the other hand, the adhesion and colonization of probiotics at the solidin-fluid phase to binding sites in the gut is the key to success of

Received: June 10, 2020

Accepted: August 18, 2020

Published: August 28, 2020 
their high performance and efficiency for human or animal health. ${ }^{6}$

In general, physical and chemical properties of powder particles, including surface and bulk properties (nano- and microscopic properties) impact various functionalities (macroscopic consequences) such as wettability, flowability, dispersibility, and stability, which involve gravity, friction, cohesion, and adhesion forces. ${ }^{15}$ Consequently, the characterization of probiotic-based product solid particles or under powder form is of great interest while being less investigated until now. Physical and chemical properties of probiotic particles in the powder state are relevant in probiotic viability and performance by controlling physicochemical (e.g., oxygen permeability and water activity), biological (e.g., surface membrane adhesion and aggregation), and/or physical (e.g., crystal formation and size, storage temperature, etc.) factors during probiotic processing, storage, and activities in the host. ${ }^{16}$ In fact, the drying techniques and delivery forms (powder beads, tablets, capsules, liquids, etc.), which depend on the physical and chemical properties of the matrix source (e.g., powder particles and dispersion) and the target (e.g., human gut), influence the probiotic performance/efficiency. ${ }^{17}$ Thus, significant changes in these fundamental parameters may considerably affect the cell viability, adhesion capacity, and growth/colonization, which could play an important role in the probiotic mechanism related to the intestinal barrier function. ${ }^{18}$ Recently, the physical and chemical characterization of mono- and multistrain probiotics at the liquid- and solid-states has been initiated. ${ }^{8}$ In such studies, powder samples have been characterized in terms of size distribution and surface properties that were assessed by probiotic lawn preparation on porous solid substrates. Besides, a coupling calorimetric technique (thermogravimetric analysis-differential scanning calorimetry (TGA-DSC)) has been successfully used for thermal profiling of powders, providing fingerprints, and predicting fundamental data for each sample. ${ }^{19}$ However, other key parameters such as the particle specific surface area or wettability also have their importance in the characterization of probiotic powder samples since this information is related to the developed surface to react with some liquid, that is, dispersing media and body fluids. Indeed, a very large surface area to volume ratio of solid particles of small size increases the possibility of accelerating the product oxidation by a large interstitial volume and an increase of the powder permeability toward water and oxygen will affect the powder stability during its storage. This parameter is sensitive to the particle size and shape, and obviously to the powder wettability. On one hand, a lower porosity or permeability reduces the moisture absorption or oxygen transfer from the ambient air inside powder particles, and on the other hand, a higher surface hydrophobicity or lower wettability limits the water absorption initially induced to powder surfaces.

This article reports on the methodology and results of an investigation performed on probiotic powder particles by using complementary techniques and approaches belonging to a series of physical chemistry tools recently reviewed. ${ }^{9}$ For this purpose, probiotic powder samples of the reference have been prepared under different solid-state forms (bulk, compressed disc, and packed-bed column) and have been characterized in terms of particle size/shape distribution and surface wettability measured by the well-known sessile drop and capillary rise techniques. ${ }^{20}$ Present results show, for the first time, the successful use of Darcy's law to quantify capillary rise experimental data and provide subsequently the powder particle bed permeability and porosity, rarely measured for probiotic quality and performance controls. A multistrain and highly concentrated probiotic powder has been chosen as the reference because it is currently used for the determination of the quality and genuineness of tests proposed for certifying probiotic consistency and quality. ${ }^{18}$ Our approach also opens a new potential way to structure-function relationship studies. We believe these probiotic powder particle properties are related to their biological activities involving cell adhesion and colonization mechanisms in model and real systems. Another interest in knowing the powder permeability or porosity to fluids is the possible correlation between the cell viability and the presence of air (oxygen) and/or moisture in inter- and intra-particle pores.

\section{RESULTS AND DISCUSSION}

The particle size and shape distribution, surface wettability, and the liquid permeability as well as the porosity of probiotic particles have been successively measured in this article on fine and bulk particles under two forms, either in a highly compressed disc or in compact porous bed, by using a standard multistrain and highly concentrated probiotic powder previously fingerprinted. ${ }^{19}$ Our goal is to pursue the physical chemistry assessment of probiotic powders under different states and with different tools, ${ }^{9}$ to identify at the microscopic scale some relevant key parameters and basic properties of solid particles that could control their macroscopic functionalities.

Powder Particle Properties. Figure 1 illustrates a typical scanning electron microscopy (SEM) image of the probiotic

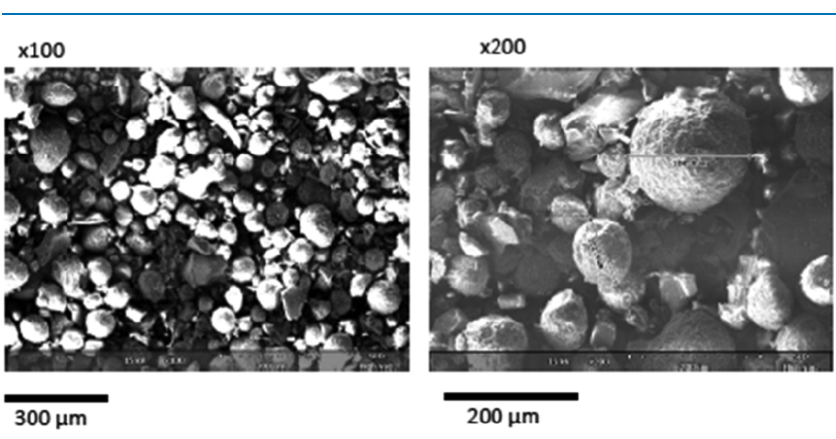

Figure 1. Scanning electron microscopy images of the probiotic powder.

powder sample, showing a diameter ranging from a few to 200 $\mu \mathrm{m}$. Powder particles appear in majority quite spherical in shape according to the images taken under well-defined conditions of experimental preparation and observation.

Figure 2 illustrates the results of the granulometry analysis. It shows the cumulative probability distributions in the size and shape of three independent probiotic particle samples. There are numerous parameters to characterize the shape of particles. We have chosen to present the convexity measurement defined as the ratio between the particle area and the convex shell estimated from a rubber band fitted on each particle. Most of the particles form convex objects and the roundness factor is around $71-72 \%$, supporting the SEM observations. The particles appear heterogeneous and contain at least two population groups around $0.3 \mu \mathrm{m}(\sim 20 \%$ in number $)$ and $3.0 \mu \mathrm{m}(\sim 80 \%$ in number $)$ mean diameters. 
A

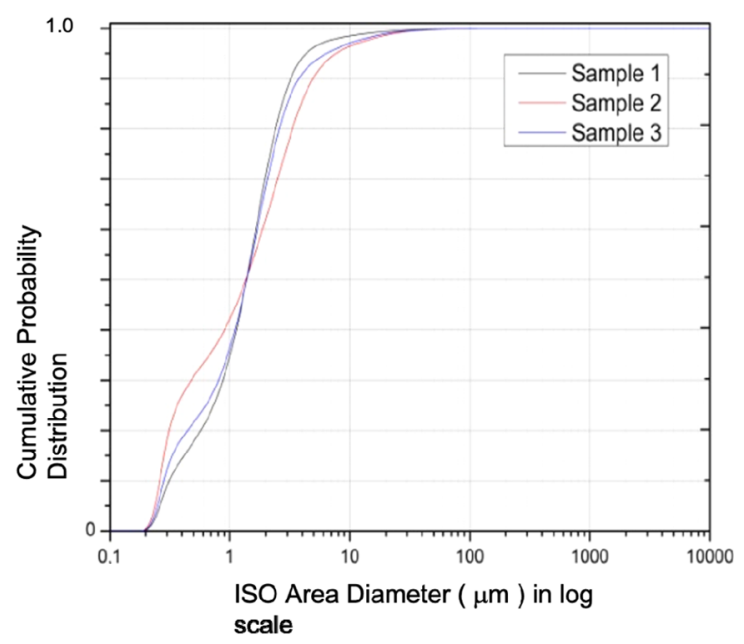

B

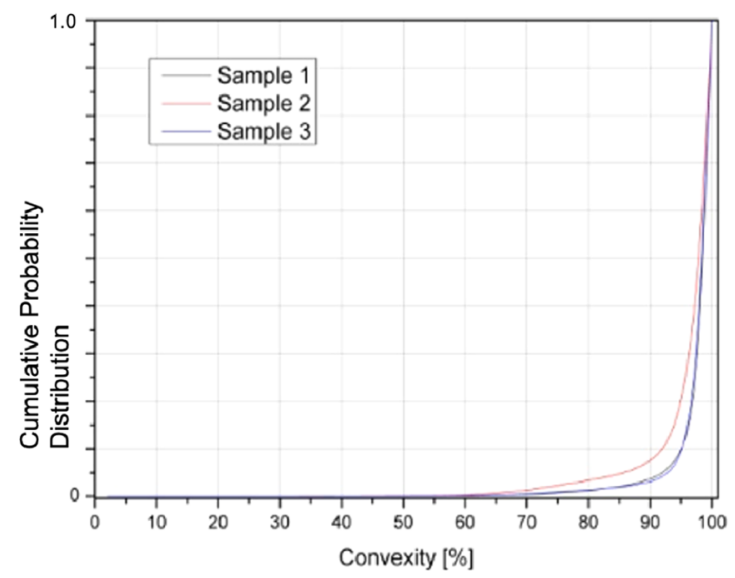

Figure 2. Probiotic particle (A) cumulative size distribution and (B) cumulative shape distribution.

Table 1 lists some particle diameter percentiles from different packages. Results show quite reproducible data characterized by a half population diameter percentile (P50) mean value of $1.830 \pm 0.017 \mu \mathrm{m}$ for the three independent packaged samples. Such statistical data represent the average of more than 100 particles in single, aggregates, and/or agglomerate forms containing various ingredients. These are typically constituted in majority by probiotic strains, mainly in cocci and rod shapes, and in minority by maltose and $\mathrm{SiO}_{2}$. These data essentially indicate that most particles have their percentile size around $1-3 \mu \mathrm{m}$. The main average particle sizes of probiotics around $1-3 \mu \mathrm{m}$ as observed through our image analysis have the same order of magnitude than the hydrodynamic diameter values $(\sim 2 \mu \mathrm{m})$ measured by dynamic light scattering (DLS) using a cumulant algorithm. ${ }^{8}$ When determined in particle-in-gas sizing mode by laser diffraction and expressed in number distribution, the same data provided a diameter $d$ around 3-4 $\mu \mathrm{m}$ (Figure S3). That means that the probiotic powder mainly contains fine or isolated cell particles and minor aggregate/agglomerate ones, as shown in the representative SEM image. The smallest particle population appearing in the cumulative distribution size $(\sim 0.3 \mu \mathrm{m})$ possibly corresponds to other minor ingredients, i.e., silicon oxide and maltose particle aggregates, commonly used in probiotic products. ${ }^{12}$ Thus, the image analyzer appears more appropriate, sensitive, and only requires the smallest amount of the sample compared to the diffraction technique for sizing probiotic powders at the solid state.

Powder Compressed Disc Properties. Equilibrium contact angles measured with all tested solvents of various physicochemical properties reach zero very quickly (after 500 $\mathrm{ms}$ ), indicating the total wettability of the compressed powder surface of the probiotic sample. Figure 3 illustrates the spreading of the solvent or Milli-Q water drops $(5 \mu \mathrm{L})$ deposited on the probiotic disc surface.

All the measured contact angles are very small, compatible with zero, which means that Zisman's critical surface tension of these probiotics is very large. ${ }^{21}$ The corresponding surfaces are therefore highly wettable. This property is, of course, very important to characterize how these probiotics interact with body fluids. Such properties are in agreement with previous results of our experiment using another preparation mode of the probiotic solid surface measured by a static advancing water contact angle method. ${ }^{8}$ There, the contact angle was immediately determined after drop deposition and a probiotic lawn was prepared on a porous filter support from a known amount of particle dispersion preparation. ${ }^{22}$ As the majority of strains of such a multistrain probiotic powder are constituted by Streptococcus thermophilus ( $>60 \%$ of total strains), ${ }^{23}$ such a result would be expected owing to the high surface affinity for water of this bacterial species, besides the presence of a hydrophilic anticake agent $\mathrm{SiO}_{2}$ in the powder. The sessile drop method combined with the compressed disc preparation under the same defined pressure $(3850$ bar) provides reproducible data and appears as a very practical tool for probiotic characterizations.

Powder-Packed-Bed Properties. Figure 4 illustrates the increase of the liquid mass squared into the powder column over time. Reproducible results for each liquid are obtained with a compaction time larger than $20 \mathrm{~min}$. Clearly two regimes can be observed in the capillary rise for each liquid: a power law behavior during the first few seconds $(2-3 \mathrm{~s})$ and then a diffusive regime for all the considered liquids. We attribute the first regime to the liquid penetration across the initial layers, which are first constituted by the filter paper followed by a small particle layer of probiotic samples. This second thin layer above the filter paper may result from the powder sample segregation due to the tapping procedure. We

Table 1. Particle Diameter Percentiles of Three Samples from Different Packages ${ }^{a}$

\begin{tabular}{|c|c|c|c|c|c|}
\hline in microns & $\mathrm{P} 10$ & $\mathrm{P} 25$ & P50 & P75 & P90 \\
\hline sample 4 & 0.325 & 0.976 & 1.840 & 2.820 & 4.110 \\
\hline sample 5 & 0.325 & 0.459 & 1.810 & 3.790 & 6.320 \\
\hline sample 6 & 0.325 & 0.859 & 1.840 & 2.980 & 4.770 \\
\hline mean $\pm S D$ & $0.325 \pm 0.000$ & $0.765 \pm 0.271$ & $1.830 \pm 0.017$ & $3.197 \pm 0.520$ & $5.067 \pm 1.134$ \\
\hline
\end{tabular}

${ }^{a} \mathrm{P}$ means the particle diameter percentile for $10,25,50,75$, and $90 \%$ of the population. 


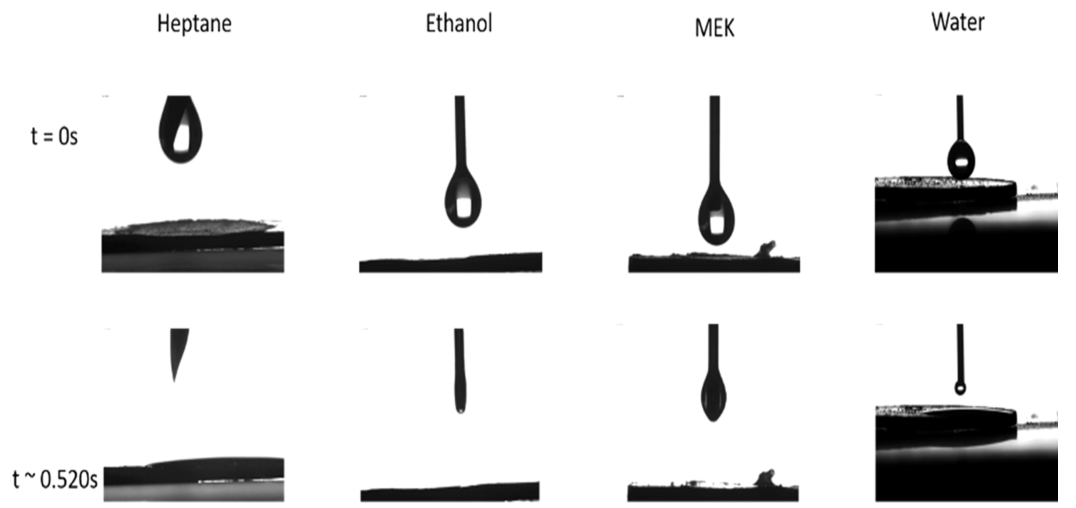

Figure 3. Spreading of different liquid drops (respectively, heptane, ethanol, methyl ethyl ketone (MEK), and water) on a compressed probiotic disc versus time (in seconds).

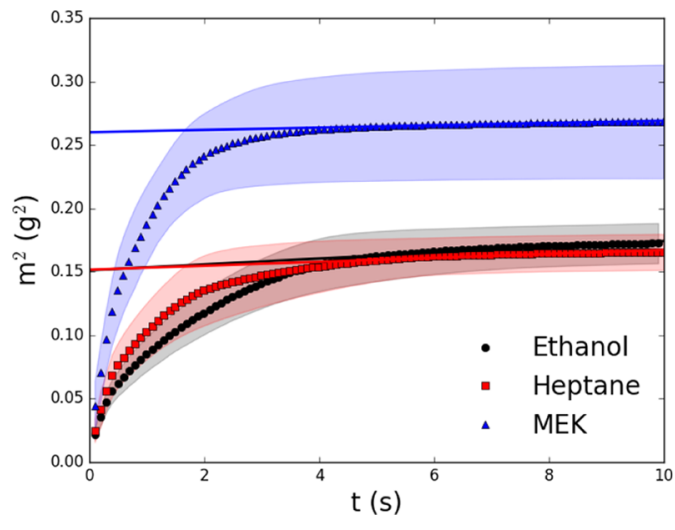

Figure 4. Increase of mass $m^{2}\left(\mathrm{~g}^{2}\right)$ during the capillary imbibition as a function of time (s) for the first 10 seconds. The error bars correspond to the statistical errors associated with four different experiments and are represented by fill areas. The three considered solvents are, respectively, ethanol (circle), heptane (square), and MEK (triangle).

have thus used the function $b \cdot t^{\alpha}+c$ to describe the first regime and $p \cdot t+q$ to describe the second one. Table 2 provides the power index $\alpha$ and the slope for each solvent.

Table 2. Fitted Parameters (Power Index and Slope) Describing the Capillary Rise in Probiotics for Ethanol, Heptane, and MEK

\begin{tabular}{lcccc}
\multicolumn{1}{c}{ liquid } & $\begin{array}{c}\text { range } \\
(\mathrm{s})\end{array}$ & $\begin{array}{c}\text { power index } \alpha \\
\left(\mathrm{m}^{2} \sim t^{\alpha}\right)\end{array}$ & $\begin{array}{c}\text { range } \\
(\mathrm{s})\end{array}$ & $\begin{array}{c}\text { slope } p\left(\mathrm{~g}^{2} / \mathrm{s}\right) \\
\left(\mathrm{m}^{2} \sim p \cdot t\right)\end{array}$ \\
heptane & $0-3$ & $0.165 \pm 0.019$ & $4-10$ & $0.00142 \pm 8 \times 10^{-5}$ \\
ethanol & $0-3$ & $0.370 \pm 0.008$ & $4-10$ & $0.00231 \pm 9 \times 10^{-5}$ \\
MEK & $0-3$ & $0.114 \pm 0.032$ & $4-10$ & $0.00091 \pm 3 \times 10^{-5}$
\end{tabular}

Clearly, in the second regime, all the considered liquids imbibe the probiotic powder with one characteristic slope. The slope of the linear fits over time periods above 3-4 s until $10 \mathrm{~s}$ to minimize evaporation effects can be easily measured. From these slopes, it is easy to get the effective radii $R_{\text {eff }}$ computed using Darcy's formula (eq 3 ), as well as the permeability $K$ determined from the slopes of different solvents using eq 3 .

Since the particle size distribution is mostly concentrated with a mean diameter around $d \sim 3 \mu \mathrm{m}$, we can use the semiempirical Kozeny-Carman from eq 4 to relate macroscopic parameters such as permeability to microscopic ones, like particle arrangement.
From our data, we get an estimate of $R_{\text {eff }}$ of the order of $350-500 \mu \mathrm{m}$, which makes sense within a probiotic tube of radius $5 \mathrm{~mm}$. Figure 5 shows the graphical presentations of the relationship between the permeability $K$ with $d=3 \mu \mathrm{m}$ (the mean diameter of the particles) and the porosity, providing porosity values between 80 and $90 \%$ for ethanol, heptane, and MEK.

The porosity is thus rather similar for all the considered liquids, turning around an average value of $85 \%$ for the porous compacted probiotic sample loaded in the glass tube. When the wettability is measured with a more porous material or powder-packed bed by using the capillary rise method, the recorded data fit well to Darcy's law that correlates permeability to the liquid flow rate, ${ }^{24}$ and not at all to Washburn's equation (Figure S3). Another advantage of Darcy's law is to provide the liquid permeability of the powder bed, or its equivalent porosity, via the Kozeny-Carman model. Considering the particle mean diameter $d=3 \mu \mathrm{m}$, this approach provides an average of powder porosity $(\sim 85 \%)$ that corresponds to the bulk porosity values $(50-80 \%)$ reported for Lactobacillus paracasei probiotic powders prepared by different drying techniques. ${ }^{25}$ These values represent the sum of the connected porosities or interparticle pores $(30-70 \%)$ and the isolated porosities or intraparticle pores $(\sim 10 \%)$ of $L$. paracasei samples.

Globally, this work reveals that the compressed disc technique for wettability measurement and the capillary rise experiments allow surface properties of probiotic powders to be characterized.

To be complete, we have also studied the capillary rise experiment using a more viscous liquid, benzyl alcohol, for which the liquid is expected to penetrate into the powder with a smaller speed. Figure 6 shows the capillary rise in the probiotic powder sample using this liquid. There are again clearly two different regimes: a power law and a linear regime. The fitted parameters are given in Table 3.

From this slope, we can estimate the effective radius $R_{\text {eff }}$ which is of the order of $400 \mu \mathrm{m}$ leading again to a porosity of $90 \%$. The fact that here we have to consider a first regime of 30 $\mathrm{s}$ is due to the viscosity of benzyl alcohol. The time required to cross the first layers of the sample has to be larger in the latter cases. Last but not the least, we have also measured the capillary rise of Milli- $Q$ water in the probiotic column. The results are presented in Figure 7 and complement our wettability measurements as described above. The associated slope for Darcy's regime is $2.95 \times 10^{-4} \pm 1.2 \times 10^{-5}\left(\mathrm{~g}^{2} / \mathrm{s}\right)$ 


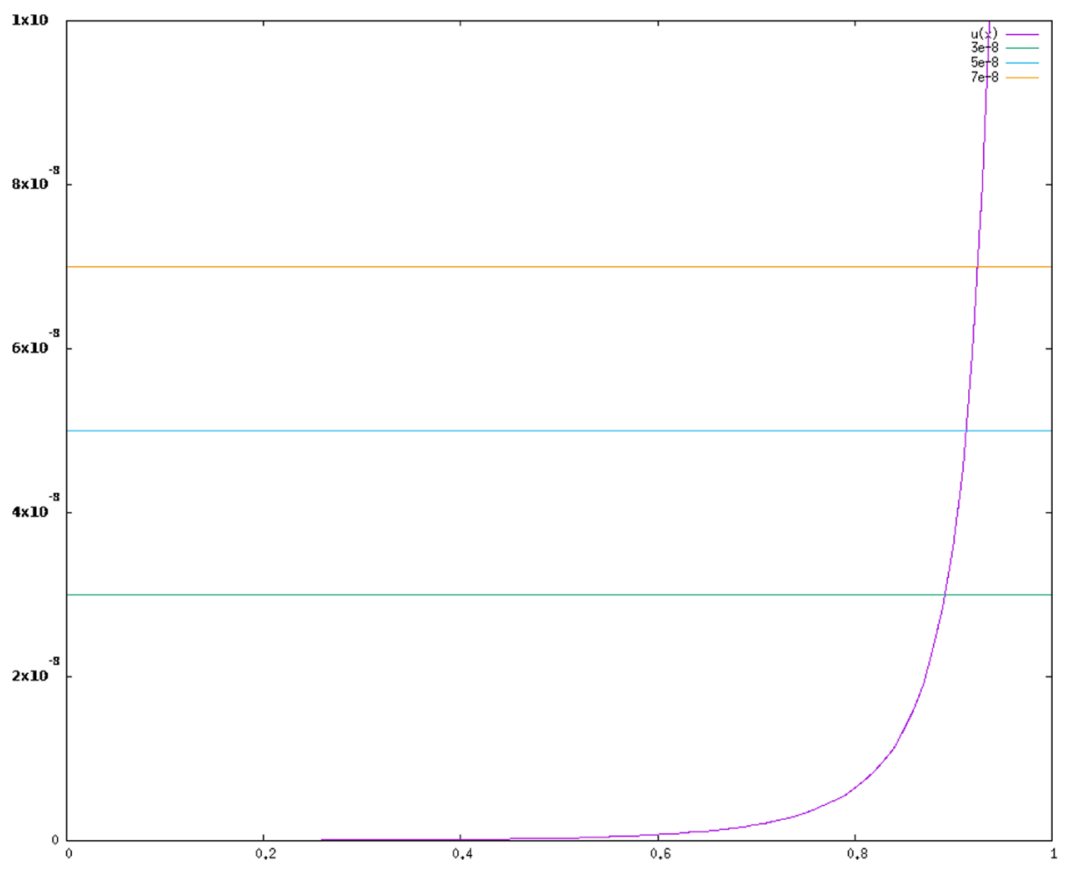

Figure 5. Permeability versus porosity determining probiotic porosity values for different solvents, from top to bottom ethanol, heptane, and MEK.

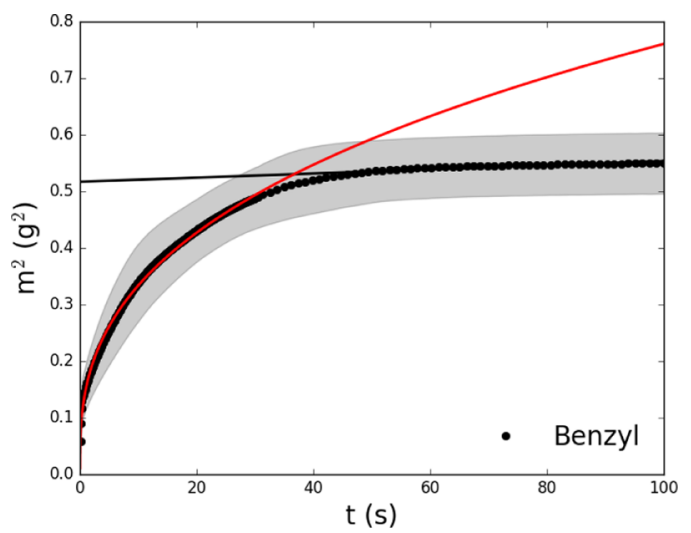

Figure 6. Capillary rise in the probiotic for benzyl alcohol: $\mathrm{m}^{2}\left(\mathrm{~g}^{2}\right)$ versus $t(\mathrm{~s})$. The error bars correspond to the statistical errors associated with four different experiments and are represented by fill areas. The two fitting curves are a power law for the first moments and a straight line for longer times. The fitted parameters are given in Table 3 .

Table 3. Fitting Parameters (Power Index and Slope) for the Benzyl Alcohol Capillary Rise

\begin{tabular}{ccccc} 
liquid & $\begin{array}{c}\text { range } \\
(\mathrm{s})\end{array}$ & $\begin{array}{c}\text { power index } \alpha \\
\left(m^{2} \sim t^{\alpha}\right)\end{array}$ & range $(\mathrm{s})$ & $\begin{array}{c}\text { slope } p\left(\mathrm{~g}^{2} / \mathrm{s}\right) \\
\left(m^{2} \sim p \cdot t\right)\end{array}$ \\
$\begin{array}{c}\text { benzyl } \\
\text { alcohol }\end{array}$ & $0-30$ & $0.366 \pm 0.005$ & $40-100$ & $0.00036 \pm 2 \times 10^{-5}$ \\
\hline
\end{tabular}

leading to an estimate for the effective radius $R_{\text {eff }}$ of $250 \mu \mathrm{m}$ and a porosity of $85 \%$.

\section{CONCLUSIONS}

In conclusion, the use of various physical chemistry techniques to characterize colloidal and surface properties of a reference probiotic powder under various solid particle forms provides coherent and reproducible results. The powder material is mainly constituted of fine and isolated particles, as well as aggregates or/and agglomerates up to $200 \mu \mathrm{m}$ rather spherical

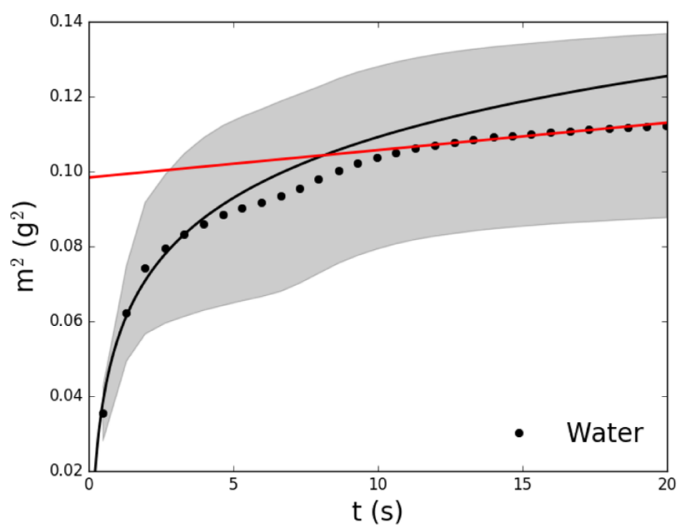

Figure 7. Capillary rise in the probiotic for water: $m^{2}\left(\mathrm{~g}^{2}\right)$ versus $t$ (ms). The error bars correspond to the statistical errors associated with four different experiments and are represented by fill areas. The two fitting curves are a power law for the first moments and a straight line for longer times.

in shape, and having a typical mean diameter about $3 \mu \mathrm{m}$. Its surface wettability measured for different solvents, either in a highly pressed disc by sessile drop or in a porous compacted solid by capillary rise shows the same trend characteristic of high critical surface tension. The powder is highly wettable. On one hand, the equilibrium contact angle measurements show complete wettability, independent of the physical and chemical characteristics of the solvents. On the other hand, the capillary rise data by far fit better to Darcy's law instead of the commonly used Washburn equation and is fully compatible with complete wettability. The use of this modeling approach leads to the possibility of assessing probiotic powder permeability and porosity. These physical properties are interesting for evaluating some performance indicators of strain stability and viability during the powder storage, as well as their adhesion capacity to the binding sites and colonization into the gut under various forms at the solid-in-fluid state. This research will open a new route to investigate the relationship 
between physical probiotic powder properties, functionalities, and performances for promoting health benefits of living species, such as the impact on the gut permeability modulation, and the cell viability due to the presence of air (oxygen) and/ or moisture in inter- and intraparticle pores.

\section{MATERIALS AND METHODS}

Probiotic Samples. Probiotic samples used in this investigation are a multistrain-based food supplement (vivomixx, reference number VSM003NM), kindly provided by Professor Claudio de Simone, as previously described and fingerprinted. ${ }^{19}$ All samples are used as received in the dry powder state, hermetically sealed in bundles containing $4.4 \mathrm{~g}$ of the material. Vivomixx is a mixture of highly concentrated (450 $\times 10^{9} \mathrm{CFU}$ ) eight bacterium strains, including $S$. thermophilus, Bifidobacterium breve, Bifidobacterium longum, Bifidobacterium infantis, Lactobacillus acidophilus, Lactobacillus plantarum, L. paracasei, and Lactobacillus delbrueckii subsp. bulgaricus. Maltose and silica dioxide ( $<5 \%$ in weight) are the other minor ingredients of this probiotic formulation. Sample fingerprints have been attested not only by the decomposition and transition thermal profiles (Figure S1) but also the black carbon and residual inorganic material content at $600{ }^{\circ} \mathrm{C}$ ( $\sim 30 \%$ in weight).

Scanning Electron Microscopy (SEM). The probiotic powder is used as provided, and its surface morphology has been observed in a Hirox SH-3000 scanning electron microscope (Hirox Company, Japan) with a voltage of 15 $\mathrm{kV}$. Prior to SEM observation, the surfaces were sputtered with gold in a Denton Desk V Sample Preparation system (Denton Vacuum L.L.C.).

Size and Shape Distribution. A granulometer 500 Nano (OCCHIO, Angleur, BE) has been used to characterize the size and shape distributions of the probiotic particles. Briefly, the powder is deposited in a plastic bag under the dispersion bell, the vacuum is launched and the plastic is then pierced, thus allowing the dispersion and the deposition of the latter on a glass slide located under the bell. Then, the bell is removed and the glass slide passes into the measuring cell, where the powder is scanned and counted (sizes and shapes) by means of optical techniques. Then, a careful image analysis is developed using algorithms according to Hart et al. ${ }^{26}$ The Occhio 500 Nano used in this study is equipped with a high resolution 6.6 mega pixel progressive scan camera and a telecentric superzoom lens to image particles with a standard resolution down to $400 \mathrm{~nm}$. This resolution is achieved by using a violet LED backlighting instead of conventional white light.

Sample Preparation. Compressed Disks. Probiotic sample disks are prepared by compressing powders into pellets with a pressure around 3835 bar corresponding to 5 tons in weight by using a manual hydraulic press (Specac). The corresponding surface is then compacted with no visible cracks and is used for the measurement of the static contact angle without chemical alteration.

Packed-Bed Columns. A probiotic powder sample (0.5 g) is weighed and loaded in the glass tube (radius $=0.5 \mathrm{~cm}$ ) closed by a filter paper at the bottom. The powder is then packed by applying 5000 taps done with a jolting volumeter model Stav II (J. Engelsmann AG, Germany) for getting reproducible measurements. This apparatus helps to compact the powder by moving up and down (tapping) under a camshaft rotation at $250 \mathrm{rpm}$. An optimum number of 5000 taps is applied for $20 \mathrm{~min}$.
Wetting Solvents. Four representative liquids with a wide range of physicochemical properties and applications have been selected (ethanol, heptane, MEK, and benzyl alcohol) for specific reasons: ethanol as the reference solvent, heptane as the low surface tension solvent, and benzyl alcohol and methyl ethyl ketone as solvents commonly used in industries. Ethanol and methyl ethyl ketone (MEK) were from Chemlab (Zedelgem, Belgium) and heptane and benzyl alcohol were provided by Sigma-Aldrich (St. Louis, MO). Table 4 lists all solvent characteristics.

Table 4. Physical Properties of Different Solvents for Wettability Measurements at $25{ }^{\circ} \mathrm{C}$

\begin{tabular}{lccc}
\multicolumn{1}{c}{ solvent } & $\begin{array}{c}\text { viscosity } \\
(\mathrm{mPa} \cdot \mathrm{s})\end{array}$ & $\begin{array}{c}\text { density } \\
\left(\mathrm{g} / \mathrm{cm}^{3}\right)\end{array}$ & $\begin{array}{c}\text { surface tension } \\
(\mathrm{mN} / \mathrm{m})\end{array}$ \\
ethanol & 1.368 & 0.789 & 21.80 \\
heptane & 0.580 & 0.684 & 19.66 \\
MEK & 0.400 & 0.800 & 23.97 \\
benzyl alcohol & 5.474 & 1.044 & 36.80 \\
\hline
\end{tabular}

Sessile Drop Method. The static contact angles were measured with a drop shape analyzer DSA 100 from Krüss (Hamburg, Germany) by depositing a $5 \mu \mathrm{L}$ drop of the liquid on the probiotic disc using the sessile drop method.

Capillary Rise Method. The capillary rise of various solvents (Table 1) into the packed-bed column with probiotic powder was monitored with a MK100 tensiometer from Krüss (Hamburg, Germany) by continuously measuring the change in the mass of the liquid penetrating the sample over time, the liquid mass $(m)$ being connected to its height $(h)$ by

$$
m=\rho \cdot \pi \cdot R_{\mathrm{eff}}^{2} \cdot h
$$

where $\rho$ represents the liquid density and $R_{\text {eff }}$ the effective radius characterizing the column imbibition.

Two mathematical models are commonly used for studying the dynamic capillary rise phenomenon monitored by the height penetration $h$ of the liquid wetting the tube or the effective tube as a function of time $t .^{27-34}$ The theoretical aspect is detailed in the available Supporting Material. Briefly, the two models are based on (1) Lucas-Washburn equation

$$
h=\sqrt{\frac{\gamma \cdot R \cdot \cos (\theta) \cdot t}{2 \cdot \eta}}
$$

where $\gamma$ denotes the surface tension, $\cos \theta$ the contact angle, $R$ the tube radius, and $\eta$ the viscosity. In this case, the porous medium is considered as a model tube of radius $R .^{35-37}$

Let us point out here that the short time behavior of the capillary rise can be affected by the friction between the movement of the air-liquid interface and the solid surface when the liquid penetrates into the tube, as illustrated by Martic et al. ${ }^{31}$ A nonuniform pore size distribution in porous media can also lead to the erroneous application of this model. ${ }^{35}$ Short time behaviors are thus always difficult to analyze in detail. (2) Darcy's law

$$
h=\sqrt{\frac{2 \cdot K \cdot \gamma \cdot t}{\eta \cdot R_{\mathrm{eff}}}}
$$

where $K$ is the so-called permeability and $R_{\text {eff }}$ the effective radius. This second model based on Darcy's law relates the 
average velocity of a liquid to the pressure gradient for the case of powders or porous media. ${ }^{36}$

By substituting $R$ with $R_{\text {eff }}$ in eq 2 , and combining it with eq 3 , one can deduce the following relation between $K$ and $R_{\text {eff }}$

$$
K=R_{\text {eff }}^{2} \cdot \cos \theta / 4
$$

In terms of mass, we thus get

$$
m^{2}=2 \cdot \pi^{2} \cdot \rho^{2} \cdot \gamma \cdot R_{\text {eff }}^{3} \cdot K \cdot t / \eta
$$

According to Darcy, fitting the data $m^{2}$ versus $t$ using a linear function $p \cdot t+\mathrm{q}$ for different liquids, we will have access to the slopes $p$ (one per liquid), which are characteristics of the porous structure. The ordinate $q$ is here to take into account the origin of time for the capillary rise.

From the kinematics of imbibition in a porous media, we can thus extract the effective radius characterizing its permeability or equivalently its porosity $(\Phi)$ through the semiempirical Kozeny-Carman approach, $, 37,38$ as follows

$$
K=\sqrt{\frac{d^{2} \cdot \phi^{3}}{180 \cdot(1-\phi)^{2}}}
$$

where $d$ represents the particle mean diameter.

\section{ASSOCIATED CONTENT}

\section{SI Supporting Information}

The Supporting Information is available free of charge at https://pubs.acs.org/doi/10.1021/acsomega.0c02728.

The theoretical aspect of the dynamic capillary rise phenomenon. Thermal profiles of the multistrain probiotic vivomixx VSM003NM by TGA-DSC: TGA curve (red), DSC curve (black), and DTG curve (blue) obtained by a $5{ }^{\circ} \mathrm{C} / \mathrm{min}$ temperature scan (Figure $\mathrm{S} 1$ ). Experimental data $(+)$ and Lucas-Washburn equation best fit $(-)$ of the ethanol capillary rise data into the vivomixx VSM003NM packed bed (Figure S2). The particle size distribution of vivomixx VSM003NM particles determined by the laser diffraction expressed in number \% (A) and in volume \% (B) (Figure S3) (PDF).

\section{AUTHOR INFORMATION}

\section{Corresponding Author}

Hary L. Razafindralambo - Gembloux Agro-Bio Tech, TERRA Teaching and Research Centre, University of Liege, B-5030 Gembloux, Belgium; 이이.orid.o000-0002-4757-7072; Phone: +32(0)81.62.21.48; Email: h.razafindralambo@ uliege.be

\section{Authors}

Mohamed A. Ali - Physics of Surfaces and Interfaces Laboratory, University of Mons, B-7000 Mons, Belgium

Giuseppina Conti - Physics of Surfaces and Interfaces Laboratory, University of Mons, B-7000 Mons, Belgium

Joël De Coninck - Physics of Surfaces and Interfaces Laboratory, University of Mons, B-7000 Mons, Belgium

Complete contact information is available at:

https://pubs.acs.org/10.1021/acsomega.0c02728

\section{Author Contributions}

M.A.A. has performed the experiments. H.L.R. has initiated and introduced the physical chemistry approach for the probiotic characterization. All the authors contributed equally to the data analysis and the writing of the paper.

\section{Funding}

This work has been supported partially by the Walloon Region, Grant numbers 1510513 and 6877.

\section{Notes}

The authors declare no competing financial interest.

\section{ACKNOWLEDGMENTS}

The authors would like to thank Professor Claudio de Simone for graciously providing samples of a multiconcentrated probiotic strain, Professor Sophie Laurent and her group for realizing the probiotic disc preparations, and Dr. Juan-Carlos Fernandez-Toledano for his appreciated help in the graphs.

\section{REFERENCES}

(1) Razafindralambo, H. Trends in Probiotic Applications; Studium Press LLC: Houston, 2018.

(2) Nguyen, H.-T.; Truong, D.-H.; Kouhoundé, S.; Ly, S.; Razafindralambo, H.; Delvigne, F. Biochemical Engineering Approaches for Increasing Viability and Functionality of Probiotic Bacteria. Int. J. Mol. Sci. 2016, 17, 867.

(3) Chaucheyras-Durand, F.; Durand, H. Probiotics in Animal Nutrition and Health. Benefic. Microbes 2010, 1, 3-9.

(4) Schisler, D. A.; Slininger, P. J.; Behle, R. W.; Jackson, M. A. Formulation of Bacillus spp. for Biological Control of Plant Diseases. Phytopathology 2004, 94, 1267-1271.

(5) FAO/WHO. Report on Joint FAO/WHO Expert Consultation on Health and Nutritional Properties of Probiotics in Food Including Power Milk with Live Lactic Acid Bacteria; FAO, 2001.

(6) Oelschlaeger, T. A. Mechanisms of Probiotic Actions-a Review. Int. J. Med. Microbiol. 2010, 300, 57-62.

(7) Cavattoni, F. Probiotic Products: Technological and Regulatory Aspects. In Trends in Probiotic Applications; Razafindralambo, H., Ed.; Studium Press LLC: Houston, TX, 2018; pp 3-12.

(8) Razafindralambo, H.; Delvigne, F.; Blecker, C. Physico-Chemical Approach for Characterizing Probiotics at the Solid and Dispersed States. Food Res. Int. 2019, 116, 897-904.

(9) Razafindralambo, H. Advances in Physical Chemistry Tools for Probiotic Characterization. In Trends in Probiotic Applications; Razafindralambo, H., Ed.; Studium Press LLC: Houston, TX, 2018; pp 50-81.

(10) Lee, Y. K.; Salminen, S. Handbook of Probiotics and Prebiotics; John Wiley \& Sons, 2009.

(11) Govender, M.; Choonara, Y. E.; Kumar, P.; du Toit, L. C.; van Vuuren, S.; Pillay, V. A Review of the Advancements in Probiotic Delivery: Conventional vs. Non-Conventional Formulations for Intestinal Flora Supplementation. AAPS PharmSciTech 2014, 15, 29-43.

(12) Sreeja, V.; Prajapati, J. B. Probiotic Formulations: Application and Status as Pharmaceuticals-A Review. Probiotics Antimicrob. Proteins 2013, 5, 81-91.

(13) Kanmani, P.; Satish Kumar, R.; Yuvaraj, N.; Paari, K. A.; Pattukumar, V.; Arul, V. Probiotics and Its Functionally Valuable Products-A Review. Crit. Rev. Food Sci. Nutr. 2013, 53, 641-658.

(14) Buckton, G. Solid-State Properties. In Pharmaceutics: The Science of Dosage Form Design, 2nd ed.; Churchill Livingstone: Edinburgh, 2002.

(15) Zafar, U.; Vivacqua, V.; Calvert, G.; Ghadiri, M.; Cleaver, J. S. A Review of Bulk Powder Caking. Powder Technol. 2017, 313, 389-401.

(16) Terpou, A.; Bosnea, L.; Kanellaki, M.; Plessas, S.; Bekatorou, A.; Bezirtzoglou, E.; Koutinas, A. A. Growth Capacity of a Novel Potential Probiotic Lactobacillus paracasei K5 Strain Incorporated in Industrial White Brined Cheese as an Adjunct Culture. J. Food Sci. 2018, 83, 723-731. 
(17) Fenster, K.; Freeburg, B.; Hollard, C.; Wong, C.; Laursen, R. R.; Ouwehand, A. C. The Production and Delivery of Probiotics: A Review of a Practical Approach. Microorganisms 2019, 7, No. 83.

(18) Razafindralambo, H.; Correani, V.; Fiorucci, S.; Mattei, B. Variability in Probiotic Formulations Revealed by Proteomics and Physico-Chemistry Approach in Relation to the Gut Permeability. Probiotics Antimicrob. Proteins 2020, 12, 1193-1202.

(19) Razafindralambo, H.; Razafindralambo, A.; Blecker, C. Thermophysical Fingerprinting of Probiotic-Based Products. Sci. Rep. 2019, 9, No. 10011.

(20) Alghunaim, A.; Kirdponpattara, S.; Newby, B. Z. Techniques for Determining Contact Angle and Wettability of Powders. Powder Technol. 2016, 287, 201-215.

(21) Butt, H.-J.; Graft, K.; Kappl, M. Physics and Chemistry of Interfaces; Wiley, 2003.

(22) Eschlbeck, E.; Bauer, S. A.; Kulozik, U. Effect of Cultivation pH on the Surface Hydrophobicity of Bacillus subtilis Spores. AMB Express 2017, 7, No. 157.

(23) Mora, D.; Filardi, R.; Arioli, S.; Boeren, S.; Aalvink, S.; de Vos, W. M. Development of Omics-based Protocols for the Microbiological Characterization of Multi-strain Formulations Marketed as Probiotics: The Case of VSL\# 3. Microb. Biotechnol. 2019, 12, 13711386.

(24) Clarke, A.; Blake, T. D.; Carruthers, K.; Woodward, A. Spreading and imbibition of liquid droplets on porous surfaces. Langmuir 2002, 18, 2980-2984.

(25) Poddar, D.; Das, S.; Jones, G.; Palmer, J.; Jameson, G. B.; Haverkamp, R. G.; Singh, H. Stability of Probiotic Lactobacillus paracasei during Storage as Affected by the Drying Method. Int. Dairy J. 2014, 39, 1-7.

(26) Hart, J. R.; Zhu, Y.; Pirard, E. Particle Size and Shape Characterization: Current Technology and Practice. EMU Notes Mineral. 2011, 7, 77-127.

(27) Lucas, R. The time law of the capillary rise of liquids. Kolloid $Z$.

1918, 23, 15-22.

(28) Washburn, E. W. The Dynamics of Capillary Flow. Phys. Rev. 1921, 17, No. 273.

(29) Hamraoui, A.; Nylander, T. Analytical Approach for the Lucas-Washburn Equation. J. Colloid Interface Sci. 2002, 250, 415421.

(30) Zhmud, B. V.; Tiberg, F.; Hallstensson, K. Dynamics of Capillary Rise. J. Colloid Interface Sci. 2000, 228, 263-269.

(31) Martic, G.; De Coninck, J.; Blake, T. D. Influence of the Dynamic Contact Angle on the Characterization of Porous Media. J. Colloid Interface Sci. 2003, 263, 213-216.

(32) Pucci, M. F.; Liotier, P. J.; Drapier, S. Capillary Wicking in a Fibrous Reinforcement - Orthotropic Issues to Determine the Capillary Pressure Components. Composites, Part A 2015, 77, 133141.

(33) Fries, N.; Dreyer, M. An Analytic Solution of Capillary Rise Restrained by Gravity. J. Colloid Interface Sci. 2008, 320, 259-263.

(34) Masoodi, R.; Pillai, K. M. Darcy's Law-based Model for Wicking in Paper-like Swelling Porous Media. AIChE J. 2010, 56, 2257-2267.

(35) Marmur, A. Penetration and Displacement in Capillary Systems of Limited Size. Adv. Colloid Interface Sci. 1992, 39, 13-33.

(36) Bear, J. Dynamics of Fluids in Porous Media; Courier Corporation, 2013.

(37) Kozeny, J. Ueber kapillare leitung des wassers im boden. Sitzungsber. Akad. Wiss. Wien 1927, 136, 271-306.

(38) Carman, P. C. Fluid Flow through Granular Beds. Chem. Eng. Res. Des. 1997, 75, S32-S48. 